\title{
SHARED SITUATION AWARENESS IN THE FLIGHT DECK-ATC SYSTEM
}

\author{
Mica R. Endsley, SA Technologies, Marietta, GA \\ R. John Hansman, MIT Intemational Center for Air Transportation, Cambridge, MA
}

Todd C. Farley, MIT Intemational Center for Air Transportation, Cambridge, MA

\section{Introduction}

New technologies and operational concept changes have been proposed for implementation in the National Airspace System (NAS). These changes include improved datalink (CPDLC) technologies for providing improved weather, traffic, Flight Object (FO) and navigation information to the pilot and controller, and new forms of automation for both the flight deck and air traffic management system (FAA, 1997). In addition, the way business is conducted in the NAS is under consideration. Increases in the discretion provided to pilots (and dispatchers in commercial airlines) are being contemplated in an effort to increase system capacity and flexibility.

New concepts of operation (e.g., Collaborative Decision Making and Free Flight) allow for more control to be given to the cockpit or airline with correspondingly greater monitoring responsibilities on the ground. In addition, new technologies and displays make possible much greater intormation flow between the ground and the cockpit and also dramatic changes in the type of information provided. Designing to support these changes suggests two integrally linked questions:

(1) What display technologies and information are needed to support desired changes in operational responsibilities?

(2) How will the changes in information availability influence the negotiation process between the cockpit and the ground?
Each of these proposed changes (both in technology and operational concept) will have a marked impact on the performance, workload and Situation Awareness (SA) of both pilots and controllers. Typically such changes are evaluated independently in terms of the effects of the proposed change on either pilot performance or ATC performance. It is proposed here, however, that in order to fully understand the effects of such changes, the joint pilot/controller system must be considered. In particular, it will be shown that not ohly do changes in one part of the system affect the operators of that system, they may also induce shifts in the need for a shared understanding of the system between pilots and controllers (shared SA), and may induce shifts in the locus of control between these two agents as the degree of shared SA itself changes.

The model in Figure 1 is presented as a framework for understanding the changes induced on the overall system by changes to one part of the system. Historically the controller possessed a high degree of control for directing the separation activities of aircraft. With this role, there was little need (or opportunity) for shared situation awareness between pilots and controllers as it was assumed that pilots would obey controller commands. Only the controller needed to have $\mathrm{SA}$ regarding this information. For activities performed primarily by the pilot (e.g. systems operations) it is assumed only the pilot needs to have this information, not the controller. 


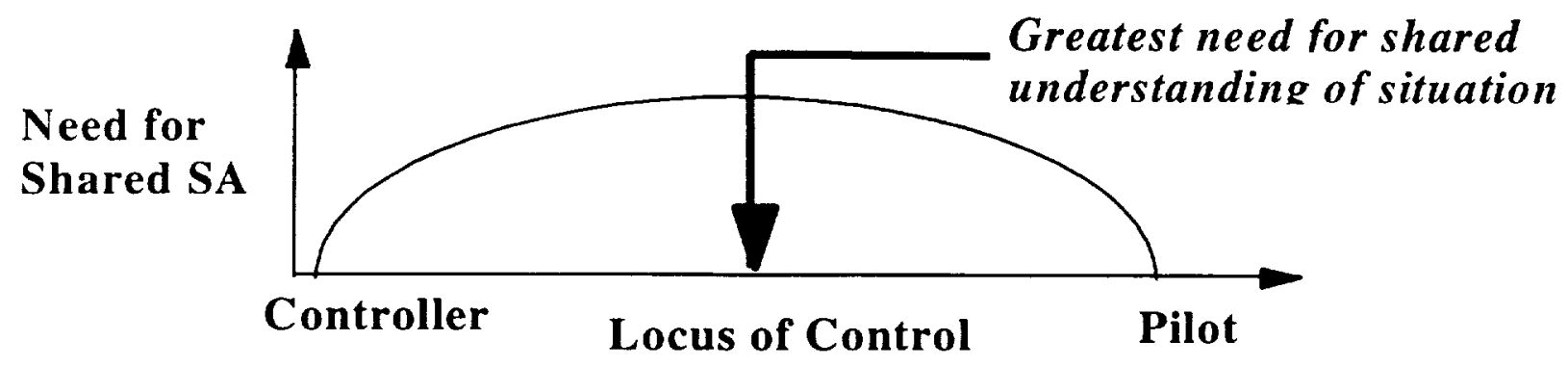

Figure 1. Shared SA and Locus of Control

As more information becomes available in the cockpit, the locus of control shifts to a middle ground. An example of this was the implementation of the TCAS collision avoidance system, where controllers had to deal with pilots interjecting unexpected actions (to the controllers) in response to TCAS alerts. In addition, TCAS was observed to change the negotiation process between controllers and pilots. With new operational concepts, such as an increase in self separation promised by some free flight advocates, it can be seen that the need for a shared common picture becomes much greater. Differences between controller SA and pilot SA may have substantial implications, resulting in problems such as increased negotiation or lack of immediate compliance to ATC commands by pilots. Therefore if pilots and controllers are going to have increasingly shared responsibilities for tasks (such as navigation, sequencing or separation activities) it will be increasingly important that they share a common picture of the environment.

\section{Analysis of Shared SA Requirements}

An analysis of shared goals and shared $S A$ requirements between pilots and controllers in the NAS was conducted to examine this issue further. This was done by comparing an $\mathrm{SA}$ requirements analysis that was conducted for en route air traffic controllers (Endsley \& Rodgers, 1994) with a similar analysis recently conducted for commercial airline pilots (Endsley, Farley, Jones, Midkiff, \& Hansman, 1998). These two sets of goals and require- ments were examined to form an understanding of (1) how these requirements overlap forming requirements for shared SA between pilots and controllers, (2) the mechanisms available to each party for acquiring SA on these factors in today's system, and (3) the implications of proposed changes to the system for shared SA and locus of control in the NAS of the future.

\section{Example of Results}

The pilot and controller goal hierarchies were analyzed and structured in order to identify areas of common or conflicting interest between pilots and controllers. Figures $2 \mathrm{a}$ and $2 \mathrm{~b}$ depict the high-level goals of pilots and controllers, respectively. At these higher levels, the goal structures were highly parallel, and there was considerable overlap between the two. For example, both shared the need to avoid conflicts such as traffic and hazardous weather as well as the need to handle perturbations such as weather and emergencies.

In examining the detailed pilot and controller SA requirements, considerable overlap was present. At the same time there were also many differences, some of which were quite subtle, even for goals which at least superficially appeared to be the same.

For instance, pilots and controllers have a common goal to maintain aircraft separation. To the pilot, conformance to ATC clearances and instructions has been the major subgoal associated with achieving aircraft separation. Assuring that aircraft are conforming to clearances, on the other hand, is seen by 


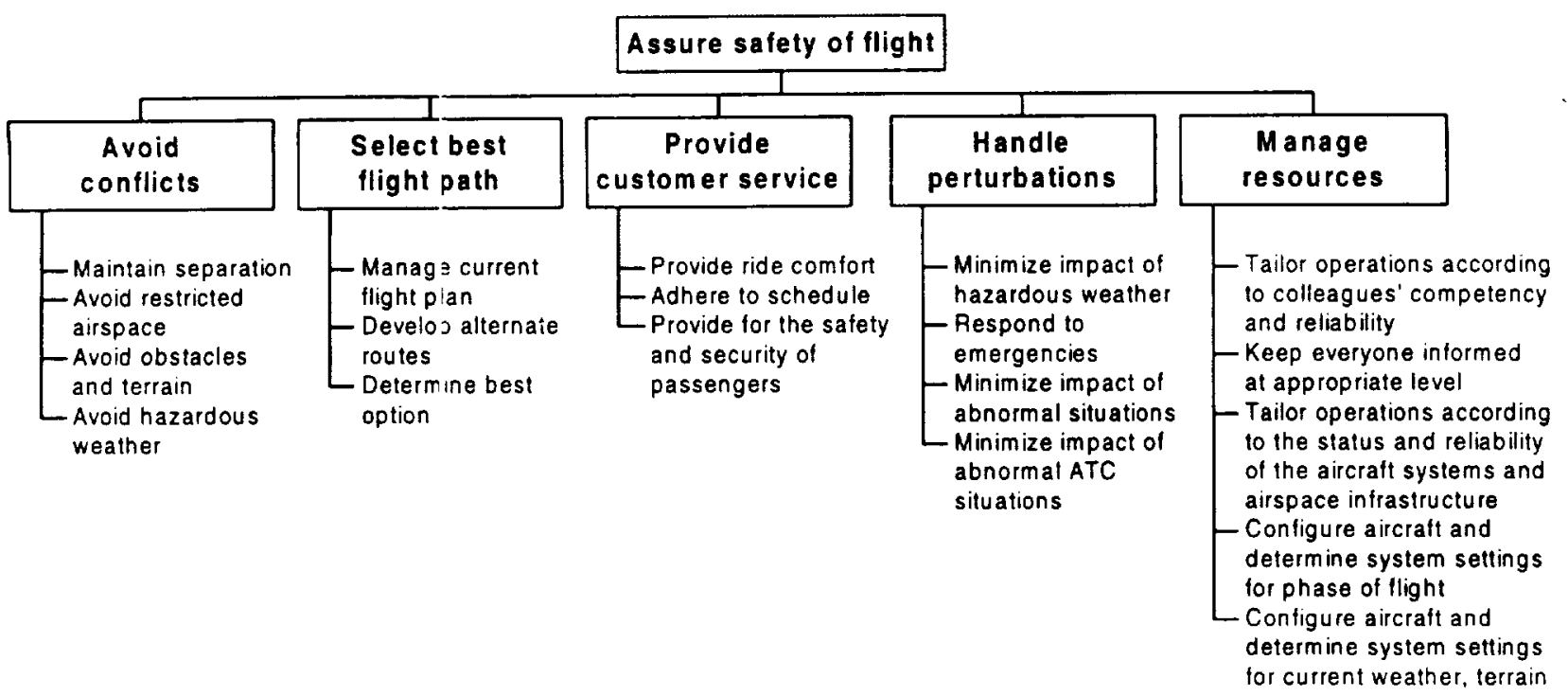

Figure 2a. Commercial Airline Pilot Top-Level Goal Hierarchy

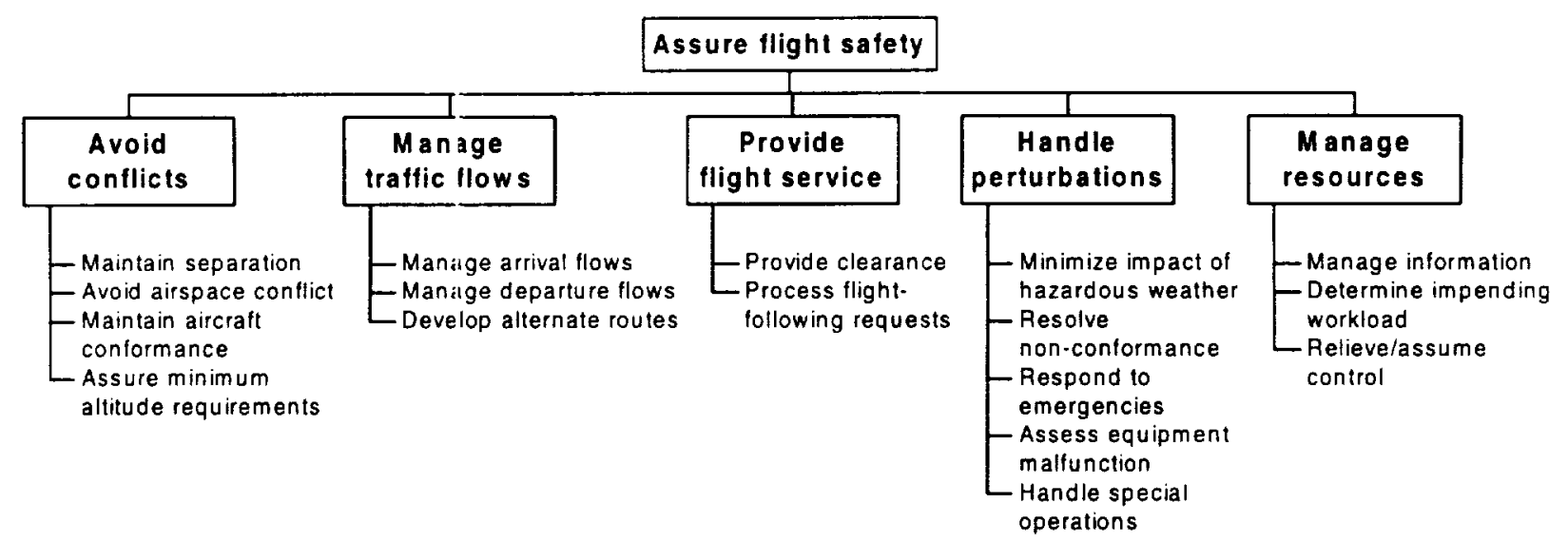

Figure 2b. En Route Air Traffic Controller Top-Level Goal Hierarchy

controllers as a separate subgoal; and one which has been found to be neglected, particularly as workload increases (Endsley \& Rodgers, 1998). In addition, if controllers do detect non-conformance to a clearance, they undertake to ascertain the reason for the nonconformance, focusing on determining the validity of the data they are receiving. Even if it is determined that non-conformance exists, the controllers may only undertake to correct the aircraft if it poses a particular problem.

Pilots on the other hand, did not represent these types of concerns in their goal breakouts. They focused on ascertaining any deviation between the ATC clearance and their current and projected flight parameters (in common with the controllers). To do this they needed several pieces of information that did not show up in the controllers' breakout, including time available to meet the clearance and the magnitude and rate of change required to meet the clearance. This represents an "information gap" between ATC and pilots. Controllers rarely communicate time available for meeting a clearance (although sometimes they may in imprecise terms convey a sense of "urgency"). Pilots are left to figure out these higher-level SA requirements on their own. Suffering from a similar information gap, controllers may often have poor information on aircraft capabilities, weather and winds that are needed for making their own assessment of 
whether an aircraft will be able to conform to a clearance as expected.

Some interesting differences are also seen in the subgoals associated with determining whether aircraft are separated. While this subgoal clearly shows up for both ATC and the aircraft pilot, they appear to meet it in very different ways. The controllers very distinctly break their decisions up into a vertical separation and horizontal separation question, with SA requirements following these divisions. Furthermore, ATC compares aircraft separations to very specific limits, as appropriate for the particular airspace. No such distinctions were present for the aircraft pilots, however. They appeared to make a single decision regarding separation, and did not appear to use any fixed limits in assessing that separation.

Finally, if it is determined that some action is needed to resolve an aircraft conflict, pilots and controllers also appear to approach this subgoal differently. While many of the decisions appear to be similar (e.g., which aircraft to maneuver, time available, which type of maneuver to use), ATC appears to adopt a much more strategic focus to its decisions while the aircraft pilot's are far more tactical. For instance, ATC includes a specific consideration of the impact of a particular maneuver on air traffic and the projected number of changes that will be necessary to bring the aircraft back to its intended flight path and destination. They also specifically consider the capabilities of the aircraft and the pilots and how many previous actions (changes to clearance) had been given to a particular aircraft. They seek to optimize the flow of the traffic overall in making their decisions. The pilots' breakouts did not reveal these type of strategic considerations, but instead relied on more immediate tactical considerations, ATC clearances and the rules built into TCAS when deciding which aircraft would maneuver and when.
Many of these differences are natural outcomes of the current division of responsibility between ATC and the cockpit, and of the information available to each. Even so, certain conflicts which exist today between these two decision makers can be traced directly back to the subtle differences in each party's interests and the different information that is available to them.

One common example is re-route negotiation, wherein the pilot typically seeks to optimize his own route while the controller typically seeks to optimize the routes of many. The supporting information available to each is often different and sometimes exclusive. Given the potential for new technologies such as datalink to share information between the air and the ground, several issues arise:

(1) How will the sharing of information between pilots and controllers affect their ability to recognize routing conflicts and opportunities?

(2) Will it increase or decrease the amount of negotiation?

(3) Will negotiations become more collaborative or more contentious?

\section{Simulator-Based Experiment}

Based on the comparative SA requirements analysis, a part-task simulator experiment was conducted to investigate the effect of sharing weather and traffic information (via air-ground datalink) between pilots and controllers in negotiating route amendments. Weather and traffic were chosen as the key conflict categories because pilots and controllers reported in the SA requirements analyses (see Figures $2 a \& b$ ) that weather and traffic are often the reason for re-routing and typically impose constraints on the available alternatives. Of particular interest in this experiment were indications of each subject's recognition of the other's constraints, his anticipation of the other's needs and/or preferences, his willingness to comply/cooperate, and his persistence in pursuing an alternate solution. 


\section{Experimental Design}

The experiment paired an air transport pilot subject with an en route air traffic controller subject in a real-time simulated air traffic environment under present-day air traffic control procedures. Test scenarios featured traffic and weather elements specifically designed to create testable responses, a performance-based measure of situation awareness (Pritchett, Hansman, \& Johnson, 1996). Testable response scenarios incorporate a hazard element (e.g., an intruder aircraft, a weather cell) that requires the subject to take action, provided s/he is aware of the situation. An appropriate action taken by the subject indicates situation awareness; naction indicates a lack of situation awareness. Subjects interacted within the simulation environment to resolve the traffic and weather conflicts. The availability of shared traffic and weather displays was manipulated as the independent variable as shown in Table 1.

Table 1. Test Matrix

\begin{tabular}{|c|c|c|}
\hline Configuration & $\begin{array}{c}\text { Traffic } \\
\text { information }\end{array}$ & $\begin{array}{c}\text { Weather } \\
\text { information }\end{array}$ \\
\hline $\begin{array}{c}\text { Without } \\
\text { datalink }\end{array}$ & $\begin{array}{c}\text { Controller } \\
\text { only }\end{array}$ & $\begin{array}{c}\text { Pilot } \\
\text { only }\end{array}$ \\
\hline With datalink & Shared & Shared \\
\hline
\end{tabular}

Because traffic display:s are standard air traffic control equipment, the controller was always provided with traffic information, even in the non-datalinked configuration. Similarly, because weather radar is standard equipment in jet aircraft, the pilot was always provided with weather information, even in the nondatalinked configuration.

Figure 3 shows the cockpit and ATC displays provided to the test subjects in the datalinked configuration, featuring both weather and traffic information on each. Weather information was depicted by six-color NEXRAD reflectivity images. Air traffic information was depicted on the controller's plan view display in a formal consistent with

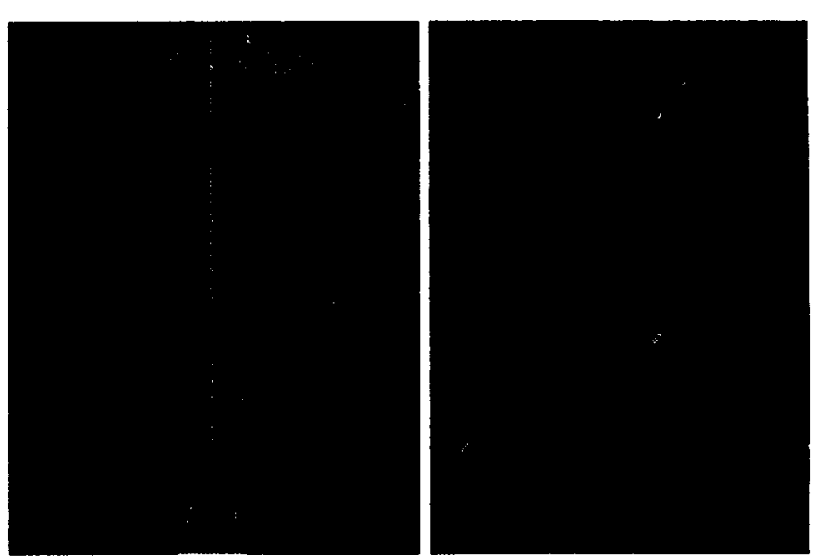

Figure 3. Cockpit map display and ATC plan view display including weather and traffic

current en route ATC equipment and included aircraft position, call sign, track, altitude, and ground speed. The pilot's cockpit display of traffic information (CDTI) depicted the position, call sign, track, relative altitude, and relative ground speed of all aircraft within 40 miles and 2600 feet in altitude.

Each pilot-controller subject pair performed three test scenarios two times, once with shared traffic and weather displays (i.e., "with datalink") and once without (i.e., "without datalink"). All scenarios took place in a high-level sector in Indianapolis Center airspace. Each scenario ran approximately ten minutes and featured between 12 and 18 aircraft transitioning the sector in the presence of convective weather activity. Those aircraft not piloted by the subject pilot were controlled by a pseudo-pilot, who also interacted with the subject controller and subject pilot via radio communication. Certain elements of each repeated scenario were changed (e.g., aircraft call signs, trajectories of non-factor traffic, etc.) in order to make the second run appear unique.

Pilot and controller situation awareness was measured using the testable response method. Radio communications were recorded and coded using a methodology adapted from Foushee, Lauber, Baetge, \& Acomb (1986). Workload measurements were taken based on the NASA Taskload Index (TLX) (Hart and Staveland, 1988). 
In order to observe pilot-controller interaction in a real-time, complex workload environment, MIT's distributed, interactive, multi-agent simulation facility was used (Amonlirdviman, et. al., 1998). The facility includes a part-task advanced cockpit simulator, a part-task en route controller workstation, a multi-aircraft pseudo-pilot station, and live voice communications between them, creating a real-time interactive air traffic environment.

\section{Preliminary Results}

Two pilot-controller teams have performed the experiment to date. Both controller subjects were Full Performance Level (FPL) ATC Specialists with an average of 10.5 years of experience, currently working the radar position at an Air Route Traffic Control Center (ARTCC). Both pilot subjects were jet transport pilots with an average of 12,850 hours.

Due to the small sample size, it is emphasized that the results presented here are preliminary. Good scientific judgment should be exercised before attempting to apply these results generally.

\section{Situation Awareness}

Each test scenario included one weather-related testable response condition and one traffic-related testable response condition. Both the pilot and controller were monitored for their awareness of each testable response condition.

Figure 4 summarizes their situation awareness with respect to weather. Pilots, having the benefit of the weather display for all test scenarios, demonstrated awareness of all of the weather-related testable response conditions. Controllers, without the benefit of a weather display in the non-datalinked configuration, demonstrated awareness of only half of the weather-related testable response conditions. When provided a weather display, however, controllers demonstrated awareness

\section{Weather SA}

Without datalink With datalink

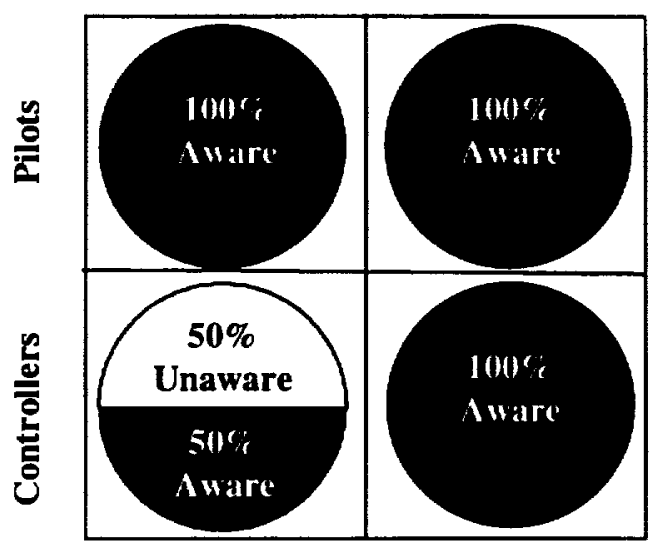

Figure 4. Pilot and controller awareness of weather-related testable response conditions.

of all of the weather-related testable response conditions.

Figure 5 summarizes pilot and controller situation awareness with respect to traffic. Pilots, without the benefit of a traffic display in the non-datalinked configuration, did not demonstrate awareness of any of the trafficrelated testable response conditions. In many cases, the controllers recognized the traffic conflict before it became a significant threat to the pilot and would either advise the pilot of the traffic or vector him accordingly. In such cases, the pilot's testable response result was labeled "ambiguous". Controllers, having the benefit of their plan view traffic display for all test scenarios, demonstrated a high level of awareness of the traffic-related testable response conditions. In some cases, controllerissued clearance amendments inadvertently resolved the traffic-related testable response conditions before they arose; such cases were labeled "ambiguous" with respect to controller situation awareness.

These results indicate that controller SA with respect to weather improves with the addition of a weather overlay to their plan view display. Similarly, the results suggest that pilot 


\section{Traffic SA}

Without datalink With datalink

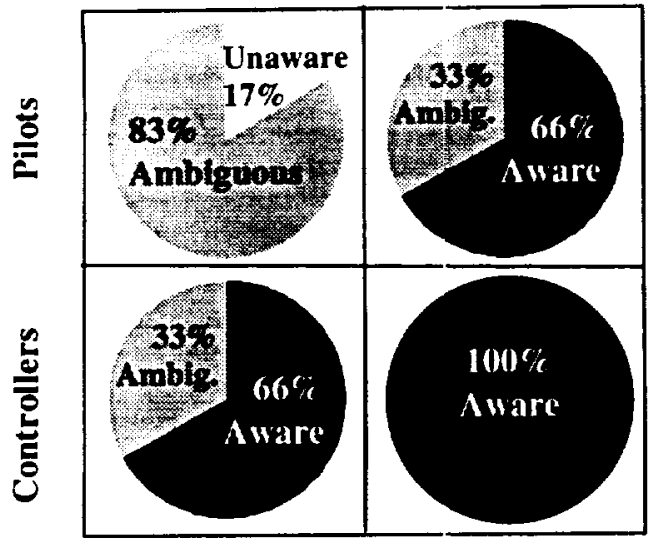

Figure 5. Pilot and controller awareness of traffic-related testable response conditions.

SA with respect to traffic improves with the addition of a CDTI.

Pilot and controller workload was measured using the NASA TL.X methodology. In general, workload was not affected in any systemic way, either individually or in a team sense.

In the twelve test sceriarios completed thus far in the study, two oferational errors ${ }^{1}$ were observed, both occurring in the nondatalinked configuration. It is important to note that the test scenarios were challenging by design. Controllers were operaing an air traffic sector other than their usual "home" sector and did not have the benefit of a conflict alert function or a D-side controller to assist them. However, the fact that both operational errors occurred in the non-datalinked environment does suggest that shared information may be beneficial in helping the controller maintain awareness with regard to separation issues.

\section{Communication and Negotiation}

All radio communication was recorded, coded by category and topic, and analyzed. Figure 6 illustrates how the transactions

\footnotetext{
${ }^{1}$ An operational error is defined as lateral separation of less
} than five miles and vertical separation of less than 1000 feet conducted over the voice channel changed with the introduction of the datalink. As shown at the left, the number of transactions between the pilot and controller decreased when the datalink was introduced. However, the number of transactions for negotiating re-route clearances increased slightly, and the number of other transactions (including traffic advisories, ride reports, etc.) decreased.

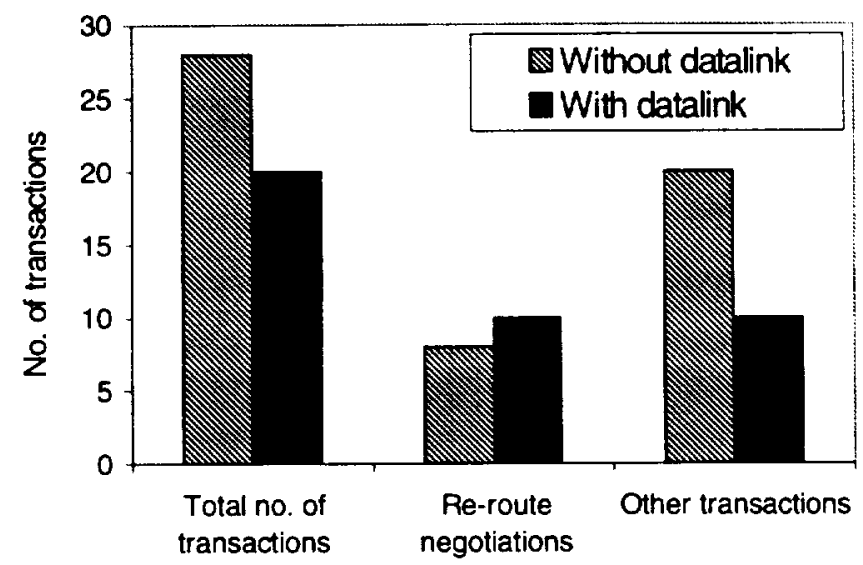

Figure 6. Voice communication transactions by topic

Figure 7 illustrates how the character of radio communications changed with the introduction of the datalink. The total number of transmissions, defined as "mic on" to "mic off", increased slightly (from 87 transmissions to 93) with the sharing of information via datalink. In analyzing these transmissions in more detail, several trends were observed. The number of pilot requests went up, consistent with the increase in re-route negotiation noted earlier. When the weather and traffic information was shared between the pilot and controller, there were four instances in which the pilot or controller voluntarily suggested a specific route amendment to the other party. This behavior was not evident in the nondatalinked configuration. Finally, controllers were much more proactive in providing weather advisories to pilots when they had the weather information overlay. 


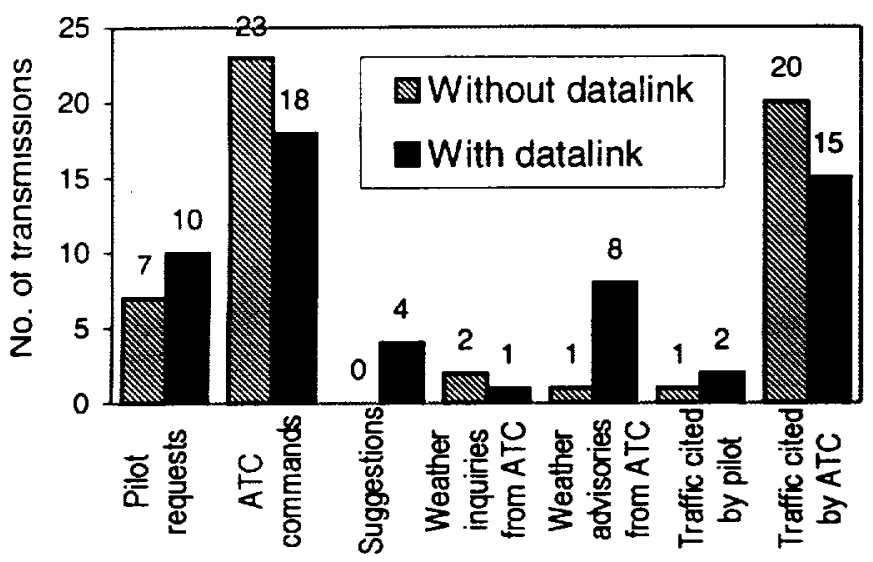

Figure 7. Voice communication transmissions by category

\section{Conclusions}

The comparison of pilots' and controllers' goals and SA information requirements revealed many parallels at the higher levels. At the more detailed, lower levels, however, subtle underlying differences were identified that lend insight into their decisions.

The preliminary experimental results indicate that situation awareness was improved when information was shared between the cockpit and the ground. Pilots' traffic situation awareness improved when traffic information was displayed; controllers' weather situation awareness improved when weather information was displayed. Operational errors were reduced in the process.

Pilots and controllers also exhibited behavioral changes. When information was shared, there were fewer requests for information, and controllers provided more frequent weather advisories to pilots. Pilots and controllers were more likely to suggest preferred route amendment alternatives to their counterparts, and re-route negotiation increased in the process.

Based on these preliminary results, it appears that shared information between flight crews and air traffic controllers may be beneficial in terms of improved situation awareness and the behaviors it seems to foster.

\section{Acknowledgments}

This work is supported by the National Aeronautics and Space Administration/Ames Research Center under grant NAG 2-716 and by TASC as part of the FAA Center of Excellence in Operations Research.

\section{References}

Amonlirdviman, K., Farley, T.C., Hansman, R.J., Ladik, J.F., \& Sherer, D.Z. (1998). "A Distributed Simulation Facility to Support Human Factors Research in Advanced Air Transportation Technology", in Proceedings of the SISO Simulation Interoperability Workshop. Orlando, FL.

Endsley, M.R., Farley, T.C., Jones, W.M., Midkiff, A.H., \& Hansman, R.J. (1998). Situation awareness information requirements for commercial airline pilots (ICAT-98-1). Cambridge, MA: MIT International Center for Air Transportation.

Endsley, M.R., \& Rodgers, M.D. (1994). Situation awareness information requirements for en route air traffic control (DOT/FAA/AM-94/27). Washington, D.C.: Federal Aviation Administration Office of Aviation Medicine.

Endsley, M.R., \& Rodgers, M.D. (1998). Distribution of attention, situation awareness, and workload in a passive air traffic control task: Implications for operational errors and automation. Air Traffic Control Quarterly, $6(1), 21-44$.

FAA (1997) National Airspace System Architecture V 3.0 (draft)

Foushee, H.C., Lauber, J.K., Baetge, M.M., \& Acomb, D.B. (1986). "Crew Factors in Flight Operations: III. The operational Significance of Exposure to ShortHaul Air Transport Operations". NASA Technical Memorandum 88322.

Hart, S.G. \& Staveland, L.E. (1988). Development of NASA-TLX (Task Load Index): Results of empirical and theoretical research. In: Human Mental Workload (P.A. Hancock and N. Meshkati (Eds.)), pp. 139-183. North-Holland: Elsevier Science.

Pritchett, A., Hansman, R.J., \& Johnson, E.N (1995). "Use of Testable Responses for Performance-Based Measurement of Situation Awareness" in (D. Garland and M. Endsley, eds.) Proceedings of the International Conference on Experimental Analysis and Measurement of Situation Awareness. Daytona Beach, FL: Embry-Riddle University Press. 